\title{
Implementation of focused ion beam (FIB) system in characterization of nuclear fuels and materials
}

\author{
A. Aitkaliyeva ${ }^{1}$, J. W. Madden, B. D. Miller, J. I. Cole \\ Idaho National Laboratory, Idaho Falls, Idaho, 83415
}

\section{Keywords}

Focused ion beam (FIB), nuclear materials, contamination, radioactivity

\begin{abstract}
Beginning in 2007, a program was established at the Idaho National Laboratory to update key capabilities enabling microstructural and micro-chemical characterization of highly irradiated and/or radiologically contaminated nuclear fuels and materials at scales that previously had not been achieved for these types of materials. Such materials typically cannot be contact handled and pose unique hazards to instrument operators, facilities, and associated personnel. Over the ensuing years, techniques have been developed and operational experience gained that has enabled significant advancement in the ability to characterize a variety of fuel types including metallic, ceramic, and coated particle fuels, obtaining insights into in-reactor degradation phenomena not achievable by any other means. The following article describes insights gained, challenges encountered, and provides examples of unique results obtained in adapting Dual Beam FIB technology to nuclear fuels characterization.
\end{abstract}

\section{Introduction}

Focused ion beam (FIB) systems have been extensively used in semiconductor industry since 1990s, mainly in modification of circuits, failure analysis, repairing of computer chips, as well as quality control. After development of dual beam FIB/scanning electron microscope (SEM) instruments, the system slowly started making its way to other scientific disciplines. Over the past decade, FIB systems have been implemented in material science and utilized for transmission electron microscope (TEM) specimen preparation. This article explores the usage of FIB systems in characterization of nuclear fuels and materials.

As in case of any sample preparation technique, FIB has advantages and disadvantages. Some of the issues associated with FIB-based sample analysis are: production of Ga-induced point defects [1], formation of intermetallic phases and Ga precipitates [2], microstructure modification [1], crystal size refinement [3], reorientation of

\footnotetext{
${ }^{1}$ Corresponding author

Idaho National Laboratory, P.O. Box 1625, M.S. 6188, Idaho Falls, ID 83415-6188, assel.aitkaliyeva@inl.gov
} 
crystallographic direction in some metals [4], curtaining in the irradiated fuel due to fissiongas-induced porosity [5] and in unirradiated systems due to differences in specimen surface topography [6-7], and differential thinning of diverse phase constituents in multi-component systems [8]. One of the surface effects that often manifests itself in metals is relaxation or modification of internal elastic stresses, frequently making thinning of TEM lamella unpredictable. Despite the challenges identified with the FIB systems, the many advantages often overshadow the list of disadvantages.

Idaho National Laboratory (INL) has pioneered the use of FIB in nuclear fuels characterization. The ability to create site-specific lift-outs allows high-resolution TEM imaging, site specific chemical mapping, and complementary local electrode atom probe (LEAP) analysis of virtually any region of interest. This is especially important for TEM examination of the nuclear fuel-cladding interaction layers formed in irradiated monolithic fuels (major focus for Reduced Enrichment for Research and Test Reactor (RERTR) program) and metal fuel-clad diffusion couples (part of research for Fuel Cycle Research and Development (FCRD) program) for which routine TEM characterization was not possible using conventional means. Conventional sample preparation techniques, particularly with complex multi-component materials, frequently require close-in, hands-on manipulation of the sample for extended periods of time. This is not feasible with highly radioactive nuclear fuels. With minimal external hands-on sample processing, specimen mass can be reduced from the bulk material to TEM lamella, atom probe tips, or small blocks for which electron backscatter diffraction (EBSD) is possible within the confines of the FIB chamber, thus significantly reducing personnel radiation exposure. The radioactivity of the extracted material used for analysis is reduced by multiple orders of magnitude, in some cases approaching natural background levels, which allows acquisition of high-quality energy dispersive spectroscopy (EDS) data with reduced background intensity. FIB preparation is relatively fast and reliable, and most importantly reproducible. In this article, we will discuss challenges faced during analysis of nuclear fuels and materials, describe equipment setup, and provide current FIB contamination levels and case studies describing advantages of implementing FIB in examination of nuclear fuels and materials.

\section{Equipment setup}

Idaho National Laboratory has two dual beam FIB/SEM microscopes implemented in characterization of nuclear fuels and materials. One is located at the INL's Electron Microscopy Laboratory (EML) capable of handling small quantities of both irradiated and unirradiated nuclear fuels (including transuranic bearing fuels) and is set up to work with sample dose rates up to $500 \mathrm{mR} / \mathrm{hr}$ gamma $(\gamma)$ at $30 \mathrm{~cm}$ (in other terms, $300 \mathrm{R} / \mathrm{hr}$ corrected beta/gamma $(\beta / \gamma)$ at contact and $15 \mathrm{R} / \mathrm{hr} \gamma$ at contact). The other tool is located at the Center of Advanced Energy Studies (CAES) and is set up to handle samples with dose rates up to $70 \mathrm{mR} / \mathrm{hr}$ corrected $\beta / \gamma$ at $30 \mathrm{~cm}$. The EML FIB system has been used to examine a number of different samples, ranging from irradiated metals and ceramics, fresh nuclear 
fuels, transuranic-bearing $(\mathrm{Np}, \mathrm{Pu}, \mathrm{Am})$ samples to irradiated fuels. As of the date of preparation of this article, the authors have not found other instances of Dual-Beam FIB preparation of irradiated fuels and transuranic samples for TEM characterization reported in the open literature.

Both instruments are FEI Quanta 3D field emission gun (FEG) FIB systems. In addition to the standard equipment, analytical attachments including EDAX EDS, EDAX wavelength dispersive spectrometer (WDS), and EDAX-TSL EBSD were installed as well as FEI Pt and C gas injectors (GIS), and Omniprobe micromanipulators on the EML FIB. For radiological work, INL has custom designed and installed a FIB enclosure with an outlet for a portable high-efficiency particulate absorption (HEPA) filter to ensure radiological safety of workers (Fig. 1). Safe radiological practices are followed to load and unload nuclear materials: i) radiological smears are taken every time the equipment is vented to ensure that no loose contamination is released from an analyzed sample, and ii) FIB contamination levels are constantly monitored and recorded, and examples of these levels are provided later in this article.

\section{Ion beam damage and sample preparation artifacts}

Basic FIB functions are: milling, deposition, and imaging. In FIB systems, material is removed via sputtering (milling), which can be understood as the removal of the outer surface layers of the material via bombardment with energetic particles. During collisions, impinging particles transfer energy to the target, which recoil and can produce other recoils. Some of these backward recoils have enough energy [9] to escape from the material and get re-deposited in the vicinity within chamber on the chamber walls, stage, detectors, and electron and/or ion beam pole pieces. The sputtering process can be considered as a collision cascade with a series of angular deflections and energy transfers between target atoms [9]. During FIB milling, implanted $\mathrm{Ga}$ ions and target atoms are removed via sputtering. The concentration of implanted Ga ions is greatest at the surface and it falls off over a distance comparable to Ga range in the target. Addition of gas injection system (GIS) allows deposition of materials, such as $\mathrm{Pt}, \mathrm{C}$, and $\mathrm{W}$, onto the surface of the target.

Energetic Ga ions can produce point defects, such as vacancies, interstitials, and defect clusters in any analyzed material. Continuous ion beam imaging and milling of a crystalline material during FIB-based sample analysis can result in structural changes, such as crystalline-to-amorphous transitions. This transformation is driven by the energy transferred to the lattice during imaging and milling and associated stopping of energetic ions in the material. The net penetration depth of an individual ion into the material is measured by the projected range, which varies depending on the elemental makeup of the substrate and the ion energy. The main parameters governing the projected range include energy and atomic number of the ion, and atomic number of the substrate, but the density, composition, and crystal structure of the substrate are important factors affecting the projected range [9]. For example, the calculated projected range of $30 \mathrm{keV}$ Ga ions in $\mathrm{U}$ is 
$8.6 \mathrm{~nm}$, while a projected range of $5 \mathrm{keV} \mathrm{Ga}$ ions in $\mathrm{U}$ is $2.7 \mathrm{~nm}$. For comparison, the projected range of $30 \mathrm{keV} \mathrm{Ga}$ ions in $\mathrm{Si}$ is $26.9 \mathrm{~nm}$ and that of $5 \mathrm{keV}$ ions is $8.1 \mathrm{~nm}$. The projected range of ions was calculated using binary Monte Carlo code Stopping and Range of Ions in Matter (SRIM) [10]. The projected range of Ga ions in any given material will give the operator an approximation of the depth of an amorphous layer created during sample preparation. Lower accelerating voltages will produce a shallower amorphous layer and therefore minimize FIB damage. Hence a low-energy cleaning ( $2 \mathrm{keV}$ and lower) step is imperative in preparation of high-quality samples with minimal surface artifacts.

As a general rule, acquisition of snapshots at any energy is preferable to continuous observation with an ion beam as it minimizes beam damage as well as sputter deposition of activated particles. The following example illustrates the difference between continuous imaging and snapshots. An individual $300 \mathrm{~ns}$ long snapshot acquired at a magnification of $5000 \mathrm{x}$ with an ion beam current of $500 \mathrm{pA}$ is equivalent to the fluence of $1.2 \times 10^{8}$ ions $/ \mathrm{cm}^{2}$, while continuous imaging for $2 \mathrm{~min}$ at the same magnification and beam current is equivalent to the fluence of $5 \times 10^{16}$ ions $/ \mathrm{cm}^{2}$. In this case the flux $\left(4 \times 10^{14}\right.$ ions $/ \mathrm{cm}^{2}$-s $)$ was kept constant and the fluence changed with increasing exposure time. By increasing or decreasing the probe current, one can manipulate the ion flux and milling rate. Here fluence is the number of ions accumulated within unit area and flux is the number of ions passing through a unit area per unit time. Prolonged exposure of the specimen to the ion beam will induce beam damage and invoke accumulation of Ga concentration in the target material, which becomes critical at the final stages of thinning of a TEM lamella.

The surface of the bulk specimen before milling is typically preserved from ion beam damage using deposition of a layer of protective material (Pt, C, etc.). Since ion beam deposition is much faster than electron beam-induced deposition, it is predominantly used for depositing this protective layer. However, one has to consider that even an individual ion beam snapshot at $30 \mathrm{keV}$ will damage the material, as has been discussed above, and region of interest will be damaged prior to the deposition of protective layer. The usage of the electron beam instead of the ion beam at the initial stage of deposition (until the deposition depth is greater than the ion penetration depth) is of high importance in surface-sensitive samples. For example in ion irradiated materials, where the penetration depth of ions can range from hundreds of $\mathrm{nm}$ to $\mu \mathrm{m}$, differentiation between damage produced with ion species and FIB Ga ions is challenging. Therefore, it is advisable to use initial electron beam induced deposition to preserve the surface of the target material, which can be followed by further ion beam assisted deposition.

One of the issues of using ion beams to prepare irradiated samples is differentiation between produced defects and irradiation-induced defects. As it can be concluded from the discussion above, energetic Ga beam produces atomic displacements and associated point defects (vacancies and interstitials) in the material. For comparison, neutron irradiation can produce, depending on the irradiation temperature and fluence: point defects, line defects (dislocation lines) [11], planar defects (dislocation loops) [12], and volume defects (voids, 
bubbles, stacking fault tetrahedral) [13-14]. The damage accumulated during FIB-based sample preparation can look very similar to the damage produced in nuclear materials irradiated to low displacements per atom (dpa) levels or lower temperatures. Figure 2 shows the microstructure of unirradiated FIB-prepared and neutron irradiated pure Fe prepared using electro-polishing in 5\% perchloric acid. The sample shown in Fig. 2(b) was irradiated to $0.19 \mathrm{dpa}$ at $300^{\circ} \mathrm{C}$ and a neutron flux of $0.95 \times 10^{14} \mathrm{n} / \mathrm{cm}^{2}-\mathrm{s}$ [15]. TEM examination confirmed the presence of defect clusters, dislocation lines, and small dislocation loops in the irradiated sample [15]. Microstructure of the unirradiated pure Fe sample, prepared in a FIB tool, is provided in Fig. 2(a) for comparison. Example shown in Fig. 2(a) indicates that $\mathrm{Ga}$ ion beam damage can be extensive enough to interfere with analysis of the sample and in some cases, depending on irradiation fluence and temperature, can be indistinguishable from neutron irradiation-induced damage. Therefore, care must be taken during FIB-based sample preparation. All sample preparation recipes used to prepare irradiated fuels and structural materials are based on standard sample preparation techniques reported elsewhere [16]. However, precautions described earlier were taken during sample preparation to eliminate FIB-based Ga damage.

\section{Radiological contamination in the FIB}

\subsection{Grid contamination}

Reduction of the specimen mass from bulk parent material to TEM lamella leads to substantial reduction of radiation dose. To give reader an idea about the activity levels of TEM lamella, the following example is provided. Three TEM specimens were prepared from a parent material $\left((\mathrm{U}, \mathrm{Pu}) \mathrm{O}_{2}\right)$ with an activity of $160 \mathrm{R} / \mathrm{hr}$ corrected beta/gamma $(\beta / \gamma)$ and $5 \mathrm{R} / \mathrm{hr} \gamma$ at contact and attached to one grid. Measurements of the grid with survey meters provided by LUDLUM Measurements, Inc. (to be referred to in the text as LUDLUM) model $3 \alpha$ and $\beta / \gamma$ hand frisker did not reveal any measurable dose rate activity level, meaning no activity above that of background could be detected. However, placement of the entire grid into LUDLUM model $3030 \alpha \beta$ sample counter and subsequent measurements revealed fixed radioactivity levels of $\sim 2000$ disintegrations per minute (dpm) $\beta / \gamma$ and $\sim 20 \mathrm{dpm}$ alpha $(\alpha)$ (approaching background radiation levels). One must note that it is not possible to precisely determine the exact location of any deposited radioactive material on the grid because the radioactivity levels provided above are obtained both from the grid itself and the attached activated TEM lamella. Nevertheless, based on the knowledge of physics of ion-solid interactions, it is most probable that the highest reading was obtained from the FIB grid itself. As it was described in detail earlier, the sample material is removed and re-deposited onto the grid, grid holder, electron beam pole-piece, and specimen stage during milling, leaving an electron transparent section on two posts. The majority of the material is typically sputtered and re-deposited in the immediate proximity of the milling region. 


\subsection{FIB chamber contamination}

The EML FIB fixed contamination levels after four years of near daily usage $(\sim 30-$ 40 hours per week) are provided in Table 1 . A variety of samples have been analyzed over this period, including non-radioactive samples, irradiated metals and ceramics, fresh nuclear fuels, transuranic-bearing $(\mathrm{Np}, \mathrm{Pu}, \mathrm{Am})$ samples, and irradiated fuels with activities ranging from $10 \mathrm{mR} / \mathrm{hr}$ corrected $\beta / \gamma$ at contact to $130 \mathrm{R} / \mathrm{hr}$ corrected $\beta / \gamma$ at contact. All contamination is fixed, which is in accordance with Department of Energy (DOE) definition means that it cannot be easily removed with a smear or light rubbing with a terrycloth towel. Figure 3 shows the inside view of the FIB chamber with approximate locations of smears and direct scans. Radiological smears were acquired from most locations, except for electron beam pole-piece and EBSD detector screen, using LUDLUM model $3030 \alpha \beta$ smear sample counter. The FIB contamination level measurements were confirmed with direct scans taken with LUDLUM model $3 \alpha$ and $\beta / \gamma$ counter. Values were initially acquired in units of counts per minute (cpm), and then converted to dpm using $10 \%$ efficiency. Fixed contamination at the CAES FIB has been detected on electron beam pole piece $\left(9 \times 10^{4} \mathrm{dpm} \beta / \gamma\right)$ only using a direct scan with LUDLUM model $3 \beta / \gamma$ counter. No loose contamination has been detected anywhere within the instrument, which is consistent with the described interaction between $\mathrm{Ga}$ ions and matter.

From the information provided above, one can see that sputtered radioactive material is deposited onto various chamber components during milling. This deposited radioactive material acts as an independent source of radiation within interior of the chamber. As of the date of preparation of this article, the measured induced radioactivity of the electron beam pole-piece is $\sim 58 \mathrm{mR} / \mathrm{hr}$ corrected $\beta / \gamma$ at contact, which was determined using direct scan method with LUDLUM model $3 \alpha$ and $\beta / \gamma$ counter. No measurable radiation levels were detected on the exterior of the FIB chamber. Typically, the thickness of FIB chamber walls is sufficient to shield the operator from the radiation exposure imposed by the radioactive samples. However, during analysis of irradiated nuclear fuel with an activity of $130 \mathrm{R} / \mathrm{hr}$ corrected $\beta / \gamma$ and $5 \mathrm{R} / \mathrm{hr} \gamma$ at contact, operator had to be shielded from the microscope using a $6.35 \mathrm{~mm}$ thick lead wall and eight lead $7.62 \mathrm{~cm}$ thick blankets. This reduced the activity on the opposite site of the wall to less than $0.5 \mu \mathrm{R} / \mathrm{hr} \gamma$ and allowed operator to safely characterize the material in the microscope without reaching an annular personal exposure limit.

The FIB system is utilized with separate vacuum pumping regions: for ion source and the column, which is maintained around $1 \times 10^{-8}$ torr with ion pumps, and for the chamber, which is typically maintained around $1 \times 10^{-6}$ torr with turbomolecular pumps backed by dry rouging pumps. Inside of the FIB chamber, a custom designed tray covers the port leading to the turbomolecular pump as a precaution for the case of undesirable sample loss and loss of debris from the sample surface. Exhaust lines leading to roughing pumps are protected with HEPA filters, which prevent release of contamination into the facility without noticeably influencing the pumping ability. Examination of the exhaust ports of the 
turbomolecular and roughing pumps after four years of operation did not reveal measurable contamination, further confirming that sputter deposition is confined to the chamber area. However, as a secondary precaution roughing pump exhaust has been connected to the laboratory suspect exhaust system since installation of the FIB system.

\section{Case studies}

In this section readers will be provided with case studies describing advantages of application of FIB tool in nuclear materials research and challenges associated with characterization of nuclear fuels. All lift-out specimens described in the following subsections were prepared using standard sample preparation technique at $30 \mathrm{keV}$ [16], which was followed by extensive cleaning at low energies $(2 \mathrm{keV})$ to eliminate FIB sample preparation-induced artifacts. The samples described in this section are representative examples of the irradiated nuclear fuels and reactor materials that were characterized since acquisition of the FIB system four years prior to the date of the article preparation. These case studies provide information on the nature of samples that were analyzed and the total activity of parent samples, which can be useful to other organizations interested in establishing facilities for evaluation of irradiated nuclear fuels in FIB tool.

\subsection{Irradiated oxide fuels}

As part of FCRD program, mixed oxides and metallic fuels have been examined in various instruments, including but not limited to SEM, FIB, and TEM. The dual beam FIB system was utilized to prepare block lift-out samples for 3D EBSD characterization of an annular mixed oxide fuel (MOX) pellet with HT-9 cladding following burn-up of 6.7\% fissions per initial metal atom (FIMA) [17]. Two cubes, with volume of approximately $25 \times 25 \times 25 \mu \mathrm{m}^{3}$ (Fig. 4(a)), were lifted-out from the parent irradiated MOX sample and placed on a FIB grid (Fig. 4(b)) [17, 18]. The FIB system allowed preparation of irradiated fuel surface for EBSD analysis, which would have been nearly impossible to achieve with in-cell mechanical polishing techniques. The dose of irradiated parent material was about $120 \mathrm{R} / \mathrm{hr}$ corrected $\beta / \gamma$ at contact, which restricts EBSD and EDS analyses by flooding the detectors with background radiation. The specimen dose was reduced to $<0.5 \mathrm{mR} / \mathrm{hr}$ in a lift-out specimen on a grid. The reduction of volume and activity of the cubes from bulk specimen lowered background radiation and allowed a comprehensive EDS and EBSD analyses to be conducted upon removal of the parent specimen from the chamber. The cubes were sectioned in series and 20 EDS/EBSD scans were acquired after each $200 \mathrm{~nm}$ slice. The ability to section these small cubes within confines of the chamber allowed specimen analysis without additional sample handling. The 3D reconstruction of the data was conducted upon completion of the experiment. Figure 4 shows EBSD reconstruction of two cubes, where $(\mathrm{U}, \mathrm{Pu}) \mathrm{O}_{2}$ matrix contains secondary (Mo-Pd-Ru-Rh-Tc) precipitates. Cube 1 in Fig. 4(a) has been exposed to temperatures of about $1500^{\circ} \mathrm{C}$ at the end of life, which is higher than that of cube $2\left(1000^{\circ} \mathrm{C}\right)[17]$. In cube 1 , two large precipitates are located on the 
grain boundaries (Fig. 4(c)) while in cube 2 inter and intra-granular precipitates are distributed throughout the cube volume (Fig. 4(d)) [18]. Even though the 3D reconstruction of the materials has been done previously [19], this was the first effort in preparation and characterization of microstructure and chemistry evolution in irradiated fuels [17]. The results of this experiment show that FIB provides a unique capability to characterize irradiated fuels despite the high background gamma and beta radiations.

The advanced gas reactor (AGR) program is focused on using a coated particle fuel with uranium oxy-carbide (UCO) in a kernel form. This kernel of fuel is embedded in four layers: 90-100 $\mu \mathrm{m}$ thick buffer layer, 35-40 $\mu \mathrm{m}$ thick inner pyrolytic carbon (IPyC) layer, 35 $\mu \mathrm{m}$ thick SiC layer, and 35-40 $\mu \mathrm{m}$ thick outer pyrolytic carbon (OPyC) layer, which serve as a fission product containment vessel [20]. Implementation of FIB system in characterization of this fuel is necessitated by the complex geometry of the fuel particle, encapsulated by porous carbon and $\mathrm{SiC}$ layers, which restricts TEM sample preparation using conventional means. Fuel kernels from the first irradiation experiment AGR-1, irradiated at the Advance Test Reactor were characterized using optical, SEM, and TEM microscopy [21]. The samples achieved $11.3 \%$ FIMA average burnup at a time-average irradiation temperature of $1070^{\circ} \mathrm{C}$ [22]. Individual fuel kernels were mechanically polished to their mid plane for microscopy characterization. Optical and SEM analysis of irradiated fuel shows that the UCO kernel forms large micron sized fission gas bubbles/pores following irradiation [23]. These large pores create FIB preparation issues such as curtaining but due to the larger micron size of these pores, FIB lift-outs can be created in lower porosity areas, which yield clean TEM lamellas. In lift-outs are prepared from high porosity areas curtaining is reduced by implementation of low angle milling. Reduction of both milling and cleaning angles by 0.5 and 2 degrees, respectively, aids in preparation of high quality specimens suitable for TEM analysis. FIB lift-outs analyzed from the UCO fuel kernels tend to show few artifacts besides curtaining and FIB-induced damage. There is little stress in the sample due to the high operation temperature of these fuels (1000$1200^{\circ} \mathrm{C}$ ). Selective area diffraction pattern (SAD) and energy dispersive spectroscopy (EDS) analyses of the fuels conducted in TEM show that the fuel is crystalline and contains three fuel phases: $\mathrm{UC}, \mathrm{UC}_{2}$, and $\mathrm{UO}_{2}$. Grains of these phases are in the single micron range. In areas where pores are present, solid fission products can be seen. Ba and Sr precipitates have been seen around the pores and are coherent with the host fuel grain. These precipitates are likely $\mathrm{BaO}$ and $\mathrm{SrO}$ due to their high affinity for oxygen. The three nanocrystalline fuel phases $\left(\mathrm{UC}, \mathrm{UC}_{2}\right.$, and $\mathrm{UO}_{2}$ ) tend to show various defects such as bubbles and dislocations. Figure 5(a) shows a secondary electron micrograph of the irradiated UCO fuel with the large porosity in the fuel and 5(b) a transmission electron micrograph of the fuel containing fission product precipitates in the fuel.

\subsection{Fresh and irradiated metal fuels}


In an effort to understand thermally activated multicomponent-multiphase diffusion and microstructural evolution in $\mathrm{Pu}, \mathrm{U}, \mathrm{Zr}$ systems that are in contact with Fe-based cladding systems, diffusion couples (DC) were examined. The diffusion couple method has an advantage that it does not restrict formation of any of the phases at thermodynamic equilibrium. However, preparation of TEM specimens from diffusion couples is extremely challenging, if not impossible, by conventional sample preparation means. Despite the fact that conventional sample preparation techniques generally provide larger electron transparent regions than FIB lift-outs, preparation of cross-sectional specimens using conventional means such as electro-polishing and broad-beam ion milling is not feasible in case of diffusion couples in part due to their fragility and in part due to radioactivity of the specimens. The width of formed fuel-cladding interaction layer containing phases of interest is typically in the order of a hundred micrometers, and preparation of these specimens for analysis requires precision that cannot be attained using electro-polishing or broad-beam ion milling but can be achieved using a FIB tool. It is common to embed such specimens in epoxy in a metallographic mount (met-mount) in order to reinforce the stability of the formed diffusion couples upon annealing and quenching in water, prevent rapid oxidation of $\mathrm{Pu}$-bearing phases, and limit spread of $\alpha$ contamination associated with the presence of $\mathrm{Pu}$ (up to $25 \mathrm{wt} \%$ ). A FIB-based technique allows preparation of site-specific lift-outs for atomic scale characterization of intermetallic phases formed within a narrow fuel-cladding interaction zone without oxidation of phases prior to TEM specimen preparation and without unnecessary spread of contamination. A U-25Pu-14Zr (in wt\%) fuel alloy in contact with $\mathrm{Fe}-12 \mathrm{Cr}$ (in wt\%) cladding was assembled into a diffusion couple and heat-treated at $700^{\circ} \mathrm{C}$ for 75 hours. The DC was then examined in the FIB and TEM. The overview of fuel and fuel-cladding interaction layer is provided in Fig. 6(a). Several lift-out were prepared from various regions within the formed fuel-cladding interaction layer (Fig. 6(b)-(d)), which allowed a thorough characterization of the formed intermetallic phases. Cross-sectional specimens show various intermetallic phases formed with fuel-cladding interaction zone. The challenge associated with these fuels is non-uniform thinning of various phases due to different milling rates of heavy and light elements. However, this issue can be easily eliminated using selective thinning and/or cleaning of regions containing individual phases within lamella until a uniform thickness throughout the specimen has been achieved. The capability to prepare cross-sectional specimens is of high importance in these specimens: it reveals subsurface features not visible in the SEM and allows characterization of fuelcladding interface. The obtained TEM results will be published elsewhere [24]. In addition, EML FIB was used to prepare TEM lamella from a 52U-20Pu-3Am-2Np-8RE-15Zr (in $\mathrm{wt} \%$ ) alloy, where RE is a rare-earth alloy of $6 \% \mathrm{La}, 16 \% \mathrm{Pr}, 25 \% \mathrm{Ce}$ and $53 \% \mathrm{Nd}$. The results of this work will also be published in separate manuscripts [25]. These efforts are the only known attempts to examine and prepare transuranic-bearing nuclear fuel samples, such as $\mathrm{Pu}$ and $\mathrm{Am}$, in the FIB. 
The primary fuel studied by the RERTR program is a U-xwt\%Mo ( $\mathrm{x}=7-10 \mathrm{wt} \%)$ metallic fuel in either a dispersion or monolithic form. Typical U-Mo dispersion fuels show a microstructure that contains crystalline fuel in the center of the fuel kernels and fuelmatrix interaction (FMI) layer that forms with the Al matrix surrounding the fuel kernels. Monolithic fuels are mostly composed of crystalline U-Mo fuel with a fuel-cladding interaction (FCI) layer forming along the cladding interface. Site-specific TEM lift-outs are very useful in characterization of these fuels. Conventional TEM sample preparation has been previously performed on the RERTR dispersion fuels [5]. Analysis of conventionally prepared samples showed that the multiphase components of the dispersion fuel lead to very little useful areas for characterization of fuel and FMI layers. For monolithic fuels, it is highly unlikely to produce a TEM sample in the FCI layer using conventional sample preparation techniques [5]. Electro-polishing randomly produces electron transparent regions around U-Mo fuel particles without precision that can be achieved in the FIB. Due to the presence of two FCI layers in irradiated monolithic fuel samples, the ability to create site-specific lift-outs is imperative for thorough characterization of irradiation effects in these samples. In addition to the need to produce site-specific specimens and fragility of the irradiated monolithic plates, FIB has the advantage of significantly reducing radioactivity of the sample, which reduces personnel radiation exposure and allows acquisition of higher quality EDS information. The site-specific sample preparation in the FIB has provided the capability to better characterize the FCI and FMI layer of the RERTR fuels. One difficulty of using the FIB on RERTR fuels is the high porosity of fuels. High density of fission gas bubbles is found along grain boundaries at lower burn-up and uniformly distributed bubbles are found throughout the fuel at higher burn-up. One of the sample preparation artifacts encountered in these fuels is heavy curtaining of the fuel due to the porosity. In instances when high porosity of fuels did not play a significant role, deposition of C vs. Pt protection layer yielded reduction of curtaining artifacts. High porosity leads to non-uniform thinning and thickness variation in porous areas and destabilization of the sample due to the high stresses in the fuel. However, sample preparation of the fuel has been achieved with sufficiently thin areas required to perform TEM analysis. Figure 7(a) shows areas in a U10Mo fuel sample where FIB cross-sections and TEM lift-outs were created in site-specific areas, Fig. 7(b) a SEM micrograph of a FIB cross-section showing fission gas bubbles populating the grain boundaries of the irradiated fuel, Fig. 7(c) TEM micrograph of the long range ordering of the bubble superlattice in the fuel, and Fig. 7(d) a high resolution TEM micrograph of the bubble superlattice.

The issues associated with preparation of porous samples, such as curtaining and non-uniform thinning, have been described above. Preparation of such specimens for TEM analysis is challenging and requires certain mitigation techniques during FIB-based sample preparation. The comparison between porosity-induced heavy and slight curtaining of the specimens is provided in Fig. 8(a) and (b), respectively. In case of high porosity of the specimen as shown in Fig. 8(a), non-uniform thinning of the specimen can leave thick areas 
not suitable for TEM analysis, while high porosity can lead to growth and coalescence of pores and in severe cases to the loss of the specimen. In preparation of such specimens, the milling angle can be reduced to minimize curtaining and maintain integrity of the sample. In our experience, reduction of milling angle from $1.5^{\circ}$ to $1^{\circ}$ or less, depending on the specimen and severity of the curtaining, during thinning and from $7^{\circ}$ to $5^{\circ}$ or less during cleaning steps substantially improves the quality of the prepared samples. In addition, selective thinning of thicker areas can be implemented to mitigate the issue.

\section{Conclusions}

Significant effort has been undertaken to establish the capability to characterize changes in irradiated nuclear fuels, both structurally and chemically, at spatial resolutions not previously obtainable due to the inherent difficulties in handling samples that conventionally would have to be prepared remotely or in glove-boxes. The challenges associated with analysis of nuclear fuels and materials have been delineated and FIB contamination levels provided. The site-specific sampling nature of the Dual-Beam FIB enables selection and detailed, high spatial resolution characterization of areas of interest, which is critical when analyzing irradiated nuclear fuels where fission product generation creates complex and spatially variant phase constituents. With these advantages come several limitations that must be understood in order to ensure correct interpretation of analysis results. Gallium ion damage can create surface amorphization, and Ga displacement damage can look similar to low-dose, low temperature neutron damage. Potentially Ga-rich compounds form in materials with low Ga solubility. These limitations can be ameliorated with careful low voltage cleaning which is particularly critical when dealing with metallic materials.

\section{Acknowledgements}

This work is supported by the U.S. Department of Energy, under DOE Idaho Operations Office Contract DE-AC07-05ID14517. Accordingly, the U.S. Government retains a nonexclusive, royalty-free license to publish or reproduce the published form of this contribution, or allow others to do so, for U.S. Government purposes.

\section{U.S. Department of Energy Disclaimer}

This information was prepared as an account of work sponsored by an agency of the U.S. Government. Neither the U.S. Government nor any agency thereof, nor any of their employees, makes any warranty, express or implied, or assumes any legal liability or responsibility for the accuracy, completeness, or usefulness of any information, apparatus, product, or process disclosed, or represents that is use would not infringe privately owned rights. References herein to any specific commercial product, process, or service by trade name, trademark, manufacturer, or otherwise, does not necessarily constitute or imply its endorsement, recommendation, or favoring by the U.S. Government or any agency thereof. 
The views and opinions of authors expressed herein do not necessarily state or reflect those of the U.S. Government or any agency thereof. 


\section{References}

[1] J. P. McCaffrey, M. W. Phaneuf, L. D. Madsen, Ultramicroscopy 87 (2001) 97-104.

[2] J. D. Casey et. al., J. Vac. Sci. Technol. B 20 (2002) 2682-2685.

[3] R. Spolenak, L. Sauter, C. Eberl, Scripta Mater. 53 (2005) 1291-1296.

[4] S. Olliges et. al, Acta Mater. 54 (2006) 5393-5399.

[5] B. Miller, J. Gan, J. Madden, J. F. Jue, A. Robinson, D. D. Keiser, J. Nucl. Mater. 424 (2012) 38-42.

[6] L. A. Giannuzzi, F. A. Stevie, Micron 30 (1999) 197-204.

[7] F. Altmann, R. J. Young, J. Micro/Nanolith. MEMS MOEMS 13(1) (2014) 011202.

[8] D. Tomus, H. P. Ng, Micron 44 (2013) 115-119.

[9] M. Nastasi, J. W. Mayer, J. K. Hirvonen, Ion-solid interactions: fundamentals and applications, Cambridge University Press, Cambridge, UK, 1996.

[10] Ziegler, J. F., Biersack, J. P., Ziegler, M. D., SRIM: the stopping and range of ions in matter, Lulu Press, Morrisville, NC, USA, 2009.

[11] B. L. Eyre, M. J. Sole, J. Nucl. Mater. 18 (1966) 314-322.

[12] A. Pochettino, M. Ipohorski, Ultramicroscopy 3 (1978) 61-67.

[13] J. H. Chute, D. G. Walker, J. Nucl. Mater. 14 (1964) 187-194.

[14] M. Hernández-Mayoral, D. Gómez-Briceño, J. Nucl. Mater. 399 (2010) 146-153.

[15] J. L. Brimhall, B. Mastel, J. Nucl. Mater. 29 (1969) 123-125.

[16] J. Mayer, L. A. Giannuzzi, T. Kamino, J. Michael, MRS Bulletin 32 (2007) 400-407.

[17] M. Teague, B. Gorman, B. Miller, J. King, J. Nucl. Mater. 444 (2014) 475-480.

[18] M. Teague, B. Gorman, Prog. Nucl. Energ. 72 (2014) 67-71.

[19] M. Schaffer, J. Wagner, Microchim Acta 161 (2008) 421-425.

[20] P. A. Demkowicz, J. D. Hunn, R. N. Morris, J. M. Harp, P. L. Winston, C. A. Baldwin, F. C. Montgomery, Preliminary Results of Post-Irradiation Examination of the AGR-1 TRISO Fuel Compacts, HTR2012-3-021.

[21] I. J. van Rooyen, D. E. Janney, B. D. Miller, P. A. Demkowicz, J. Riesterer, Nucl. Eng. Des. 271 (2014) 114-122

[22] I. J. van Rooyen, T. M. Lilo, Y. Q. Wu, J. Nucl. Mater. 446 (2014) 178-186.

[23] I. J. van Rooyen, D. E. Janney, B. D. Miller, P. A. Demkowicz, J. Riesterer, Electron microscopic evaluation and fission product identification of irradiated TRISO coated particles from the AGR-1 experiment, a preliminary review, Paper HTR 2012-3-023, in: Proceedings of the HTR 2012, Tokyo, Japan, October 28-November 1, 2012.

[24] A. Aitkaliyeva, J. Madden, B. Miller, T. P. O'Holleran, J. R. Kennedy, manuscript under preparation.

[25] D. Janney, J. R. Kennedy, J. Madden, T. P. O’Holleran, J. Nucl. Mater. 448 (2014) 109-112. 


\section{Captions}

Figure 1. Images of the FIB setup.

Figure 2. TEM micrographs of (a) unirradiated Fe after milling in a FIB tool and (b) Fe irradiated to $0.19 \mathrm{dpa}$ at $300 \mathrm{C}$ with neutrons. (b) is reprinted from Ref. [15].

Table 1. EML FIB contamination levels. * indicates position of EBSD detector in retracted and inserted positions.

Figure 3. Image of the FIB chamber. White arrows and numbers correspond to approximate direct frisk and smear positions.

Figure 4. SEM micrographs of a MOX fuel (a) prior to lift-out and (b) after lift-out and mounting on FIB grid. EBSD reconstruction of the distribution of metallic precipitates in cubes 1 and 2 are shown in (c) and (d), respectively. Metallic (Mo-Pd-Ru-Rh-Tc) precipitates are shown in indigo and different grains of $(\mathrm{U}, \mathrm{Pu}) \mathrm{O}_{2}$ matrix are depicted by orange, royal blue, aquamarine, and violet colors to illustrate the difference in grain orientations. Reprinted from ref. [17].

Figure 5. (a) SEM micrograph of the irradiated UCO fuel showing the large micron sized porosity in the fuel and (b) TEM micrograph of the $\mathrm{UO}_{2}$ fuel containing fission product precipitates in the fuel. White arrows denote $\mathrm{BaO} / \mathrm{SrO}$ precipitates.

Figure 6. SEM micrograph of (a) the fuel, cladding, and formed interaction zone (denoted by two white dashed lines), and (b)-(d) TEM lamella prepared from within fuel-cladding interaction zone, with exposed various intermetallic phases, prior to thinning to electron transparency. Red rectangular boxes in (a) indicate approximate position of lift-outs from fuel-cladding interaction zone identified by two white dashed lines.

Figure 7. (a) Locations of where FIB lift-outs and cross-sections were site specifically prepared, (b) SEM micrograph of a FIB cross-section with fission gas bubbles populating the grain boundaries of the fuel, (c) TEM micrograph of the long range ordering of the bubble superlattice in the fuel, and (d) high resolution TEM micrograph of the bubble superlattice. Reprinted from ref. [5].

Figure 8. TEM micrographs illustrating (a) severe curtaining associated with the presence of high porosity in the specimen and (b) slight curtaining due to the presence of two pores. 

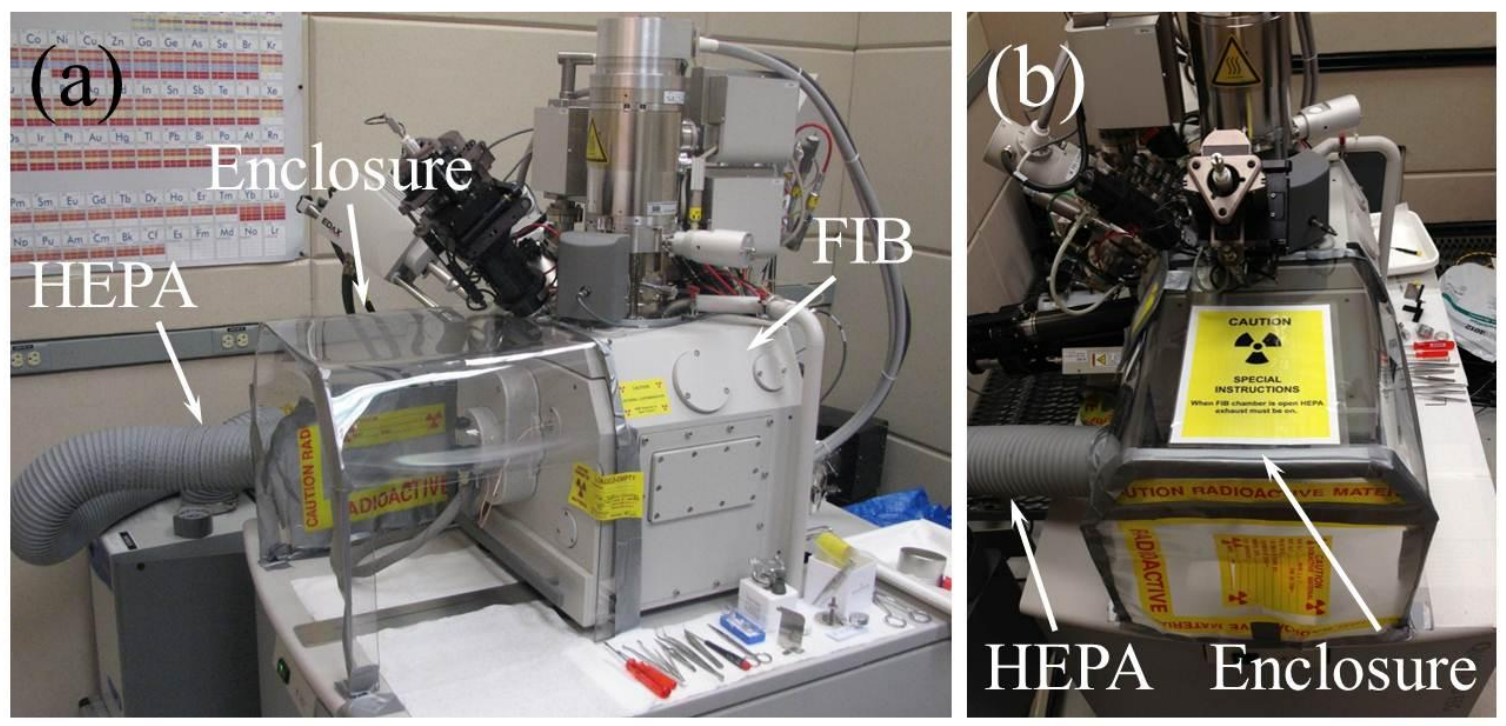

Fig. 1. 

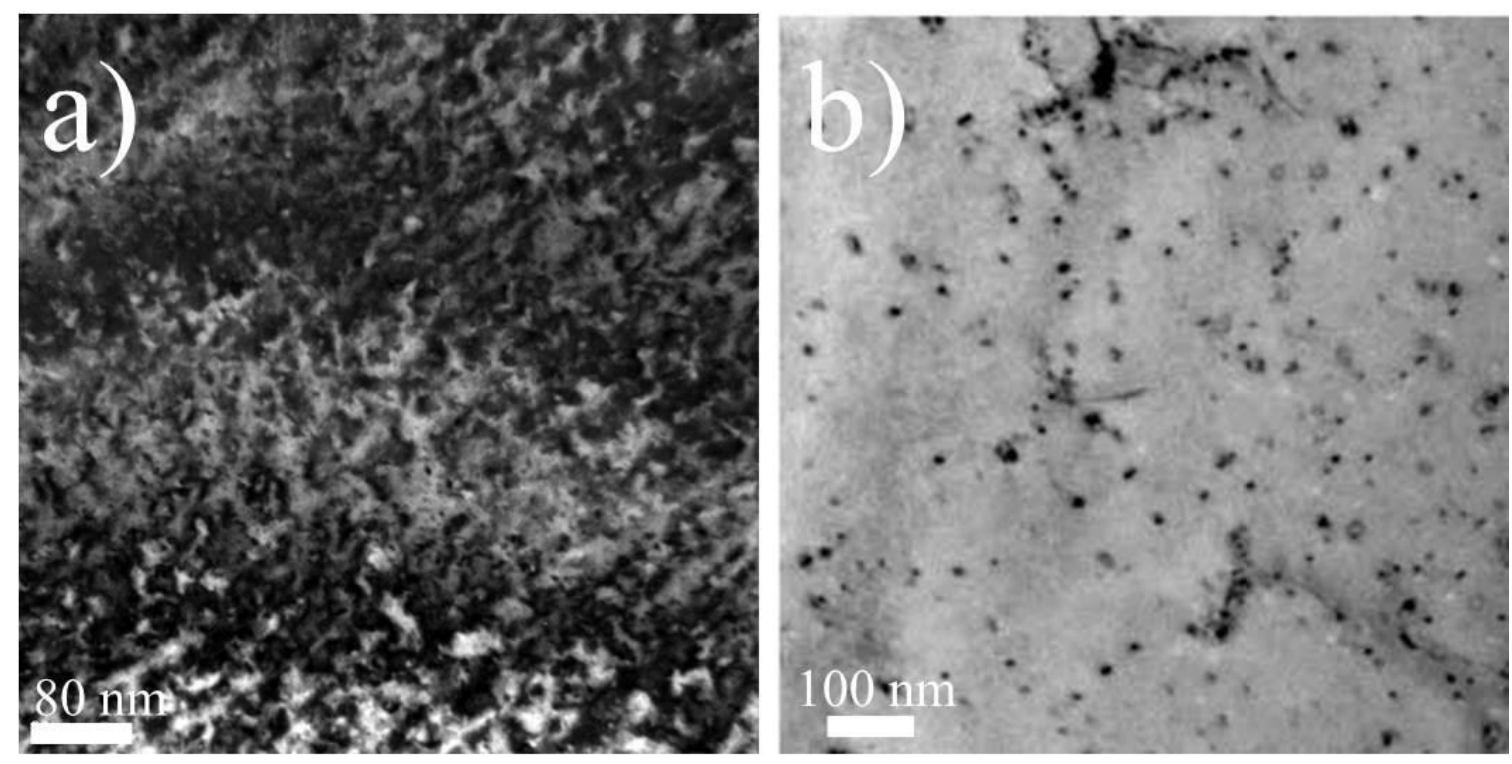

Fig. 2. 


\begin{tabular}{|c|c|c|c|c|c|c|}
\hline \multirow{2}{*}{$\begin{array}{c}\text { Scan } \\
\#\end{array}$} & \multirow{2}{*}{ Scan location } & \multicolumn{2}{|c|}{$\begin{array}{c}\text { Contamination levels in } \\
\text { dpm }\end{array}$} & \multirow{2}{*}{ Reading type } & \multirow{2}{*}{ Sample counter } & \multirow{2}{*}{$\begin{array}{l}\text { Distance to } \\
\text { specimen } \\
\text { surface, cm }\end{array}$} \\
\hline & & $\alpha$ & $\begin{array}{c}\text { corrected } \\
\beta / \gamma\end{array}$ & & & \\
\hline 1 & $\begin{array}{l}\text { Electron beam } \\
\text { pole-piece }\end{array}$ & $4.7 \times 10^{6}$ & $7 \times 10^{5}$ & Direct & $\begin{array}{c}\text { LUDLUM } 3 \alpha \\
\& \beta / \gamma\end{array}$ & 1 \\
\hline 2 & $\begin{array}{l}\text { EBSD detector } \\
\text { screen }\end{array}$ & $5 \times 10^{3}$ & $3.5 \times 10^{5}$ & Direct & $\begin{array}{c}\text { LUDLUM } 3 \alpha \\
\& \beta / \gamma\end{array}$ & $18 / 2^{*}$ \\
\hline 3 & Left chamber wall & 0 & $2 \times 10^{5}$ & Direct \& Smear & $\begin{array}{l}\text { LUDLUM } 3 \alpha \\
\& \beta / \gamma, 3030 \alpha \beta\end{array}$ & 18 \\
\hline 4 & Back chamber wall & 0 & $4 \times 10^{4}$ & Direct \& Smear & $\begin{array}{l}\text { LUDLUM } 3 \alpha \\
\& \beta / \gamma, 3030 \alpha \beta\end{array}$ & 18 \\
\hline 5 & $\begin{array}{l}\text { Right chamber } \\
\text { wall }\end{array}$ & 0 & $1.5 \times 10^{4}$ & Direct \& Smear & $\begin{array}{l}\text { LUDLUM } 3 \alpha \\
\& \beta / \gamma, 3030 \alpha \beta\end{array}$ & 18 \\
\hline 6 & Chamber floor & 0 & $12 \times 10^{4}$ & Direct \& Smear & $\begin{array}{l}\text { LUDLUM } 3 \alpha \\
\& \beta / \gamma, 3030 \alpha \beta\end{array}$ & 16 \\
\hline 7 & Specimen stage & $2 \times 10^{3}$ & $3 \times 10^{4}$ & Direct \& Smear & $\begin{array}{l}\text { LUDLUM } 3 \alpha \\
\& \beta / \gamma, 3030 \alpha \beta\end{array}$ & 5 \\
\hline
\end{tabular}

Table 1. 


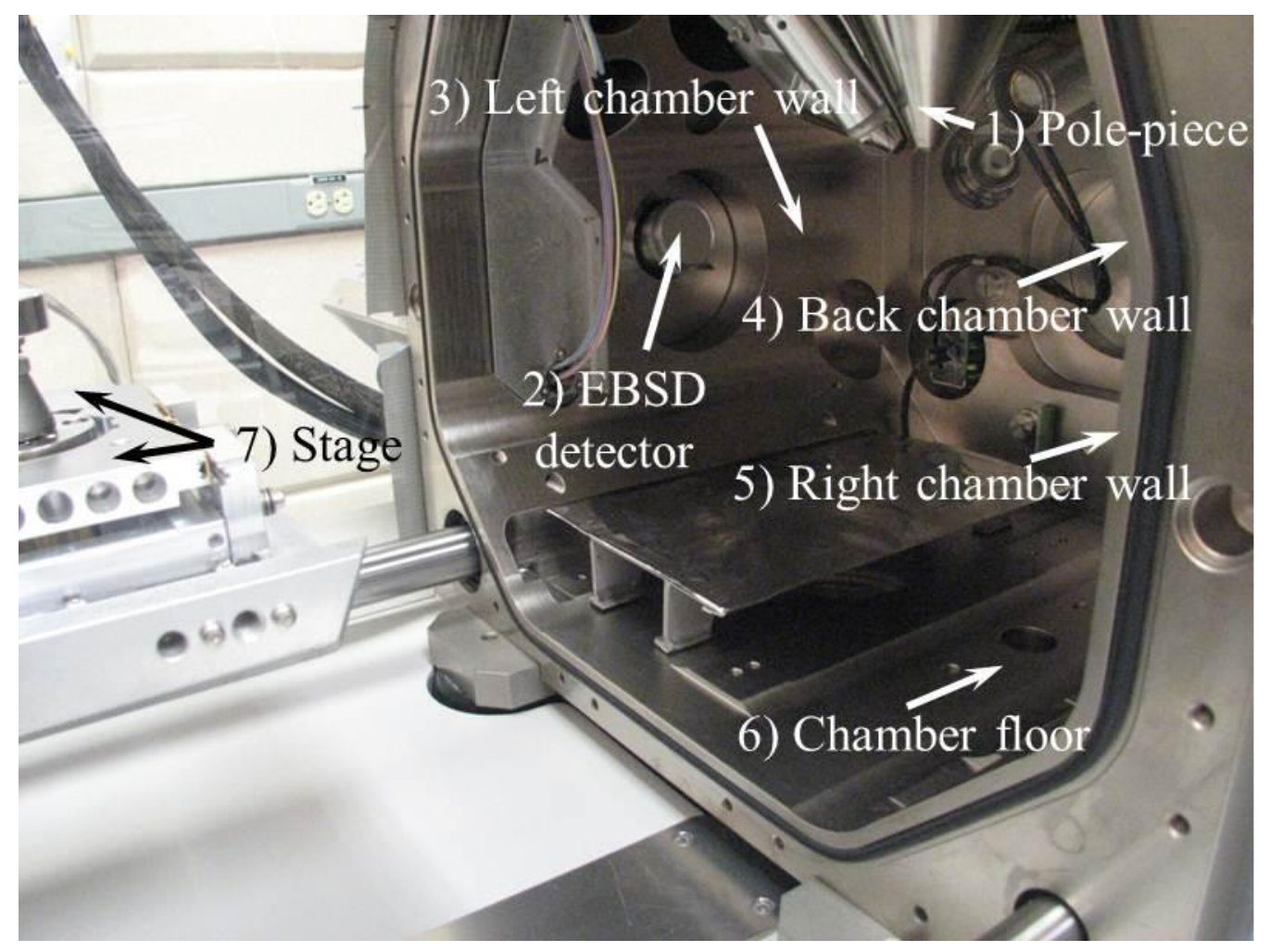

Fig. 3. 

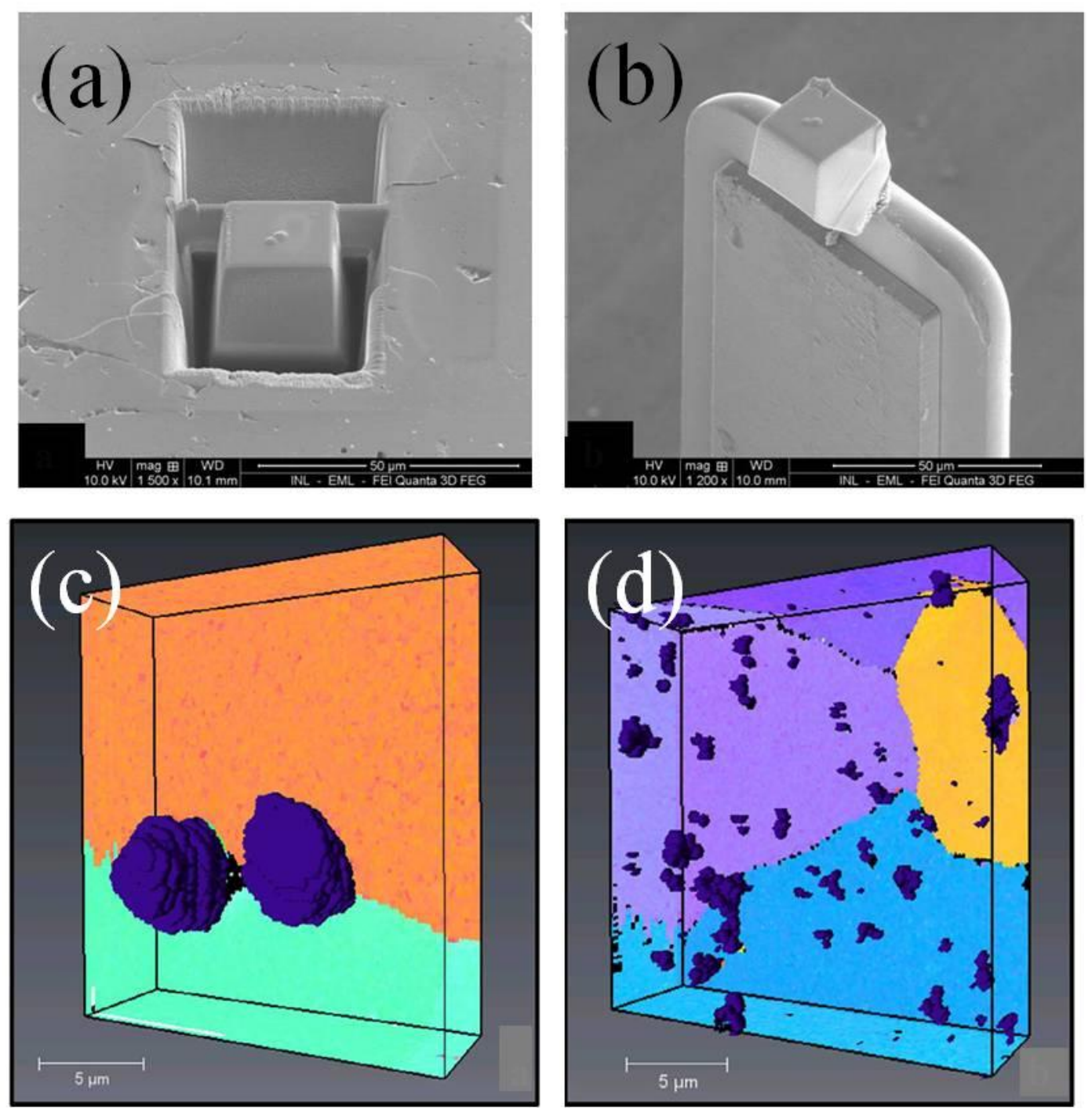

Fig. 4. 

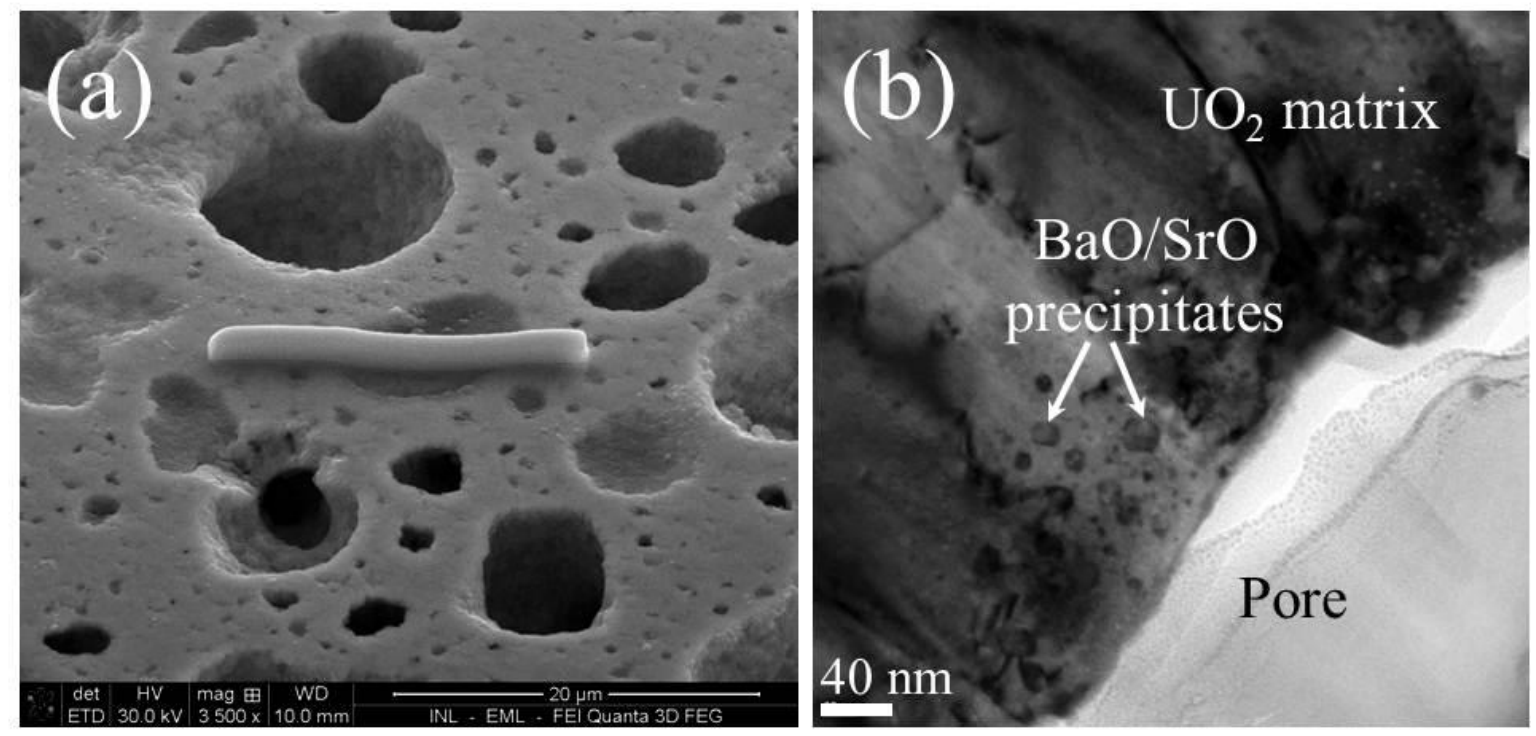

Fig. 5. 

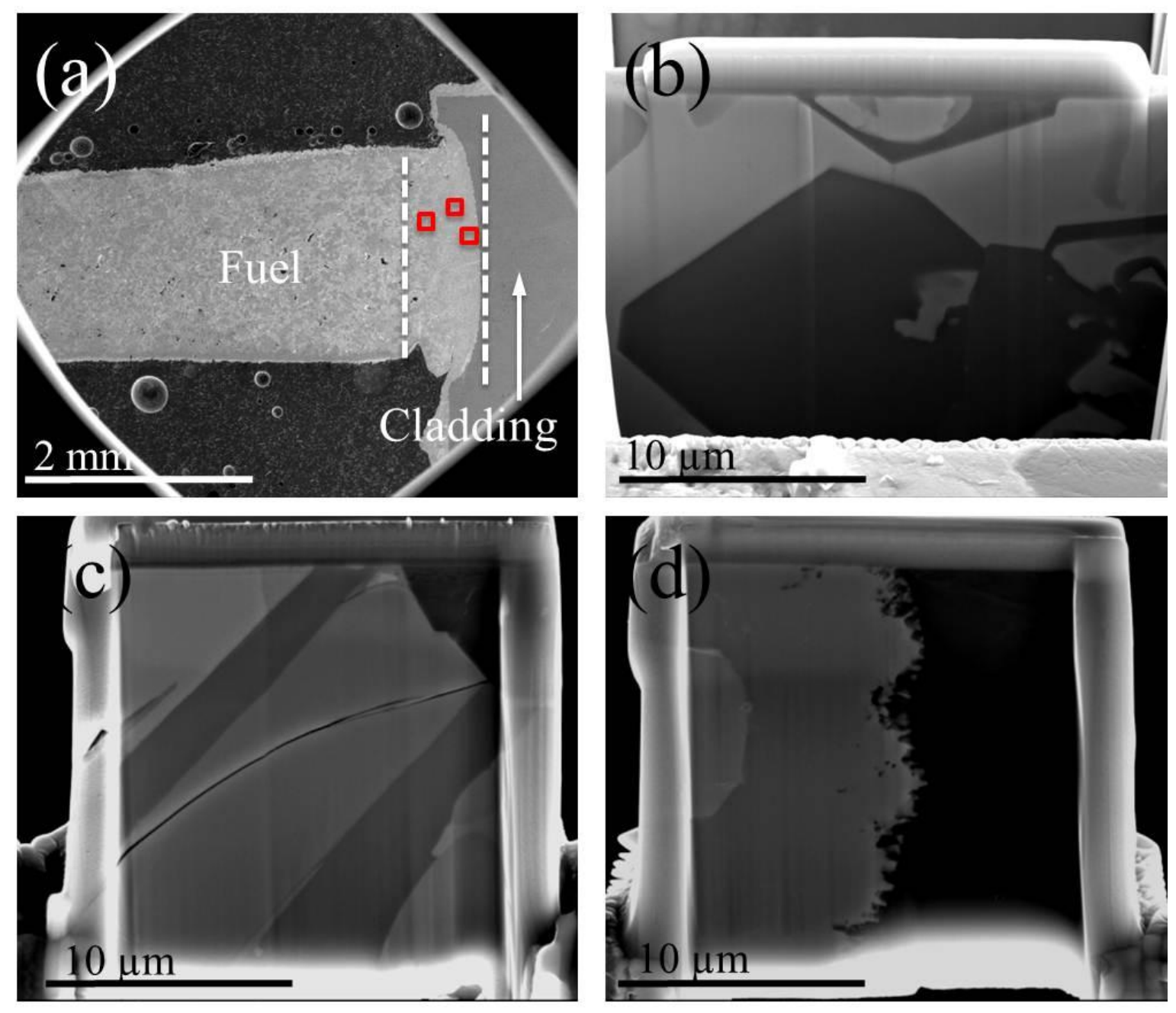

Fig. 6. 

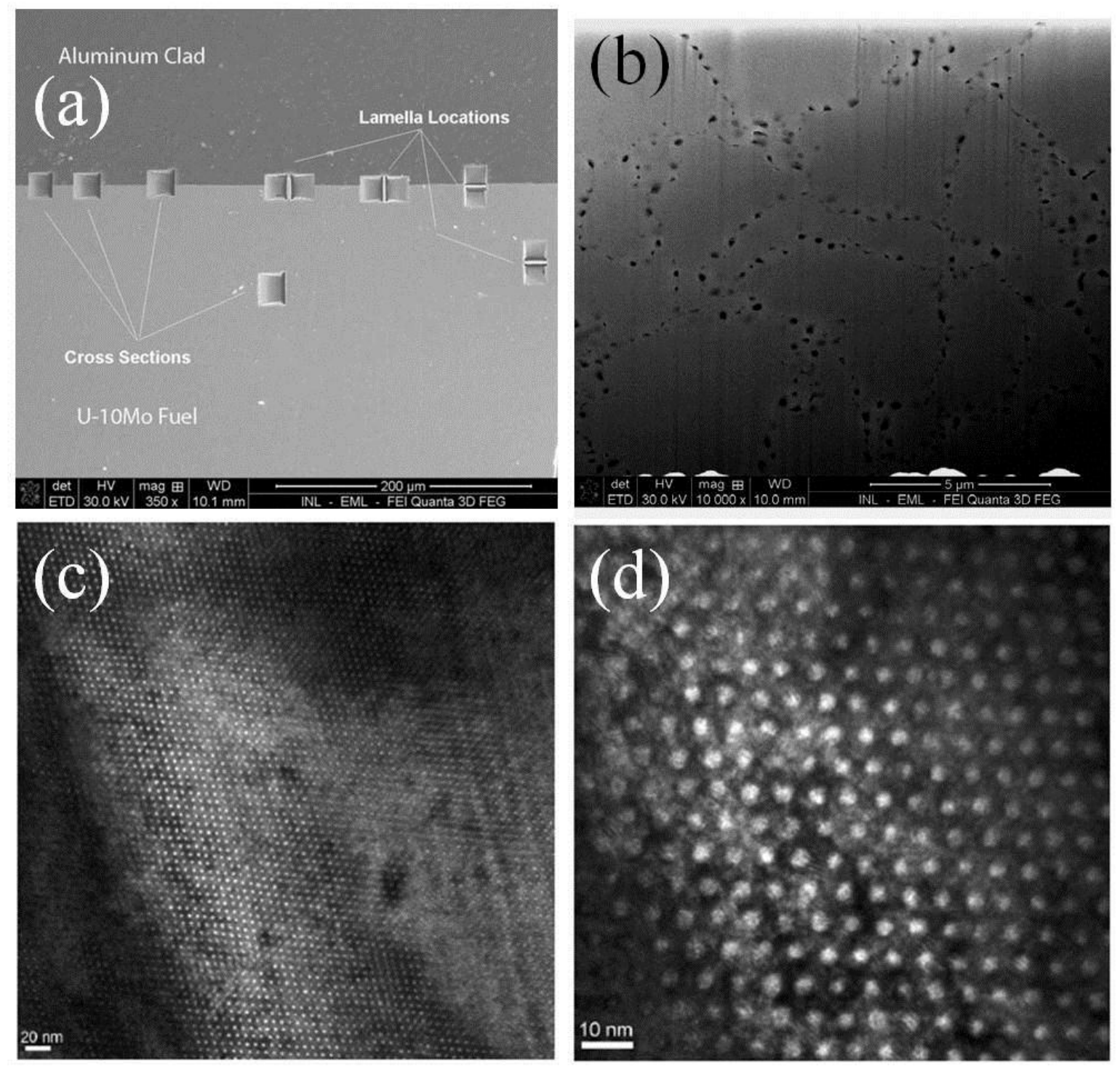

Fig. 7. 


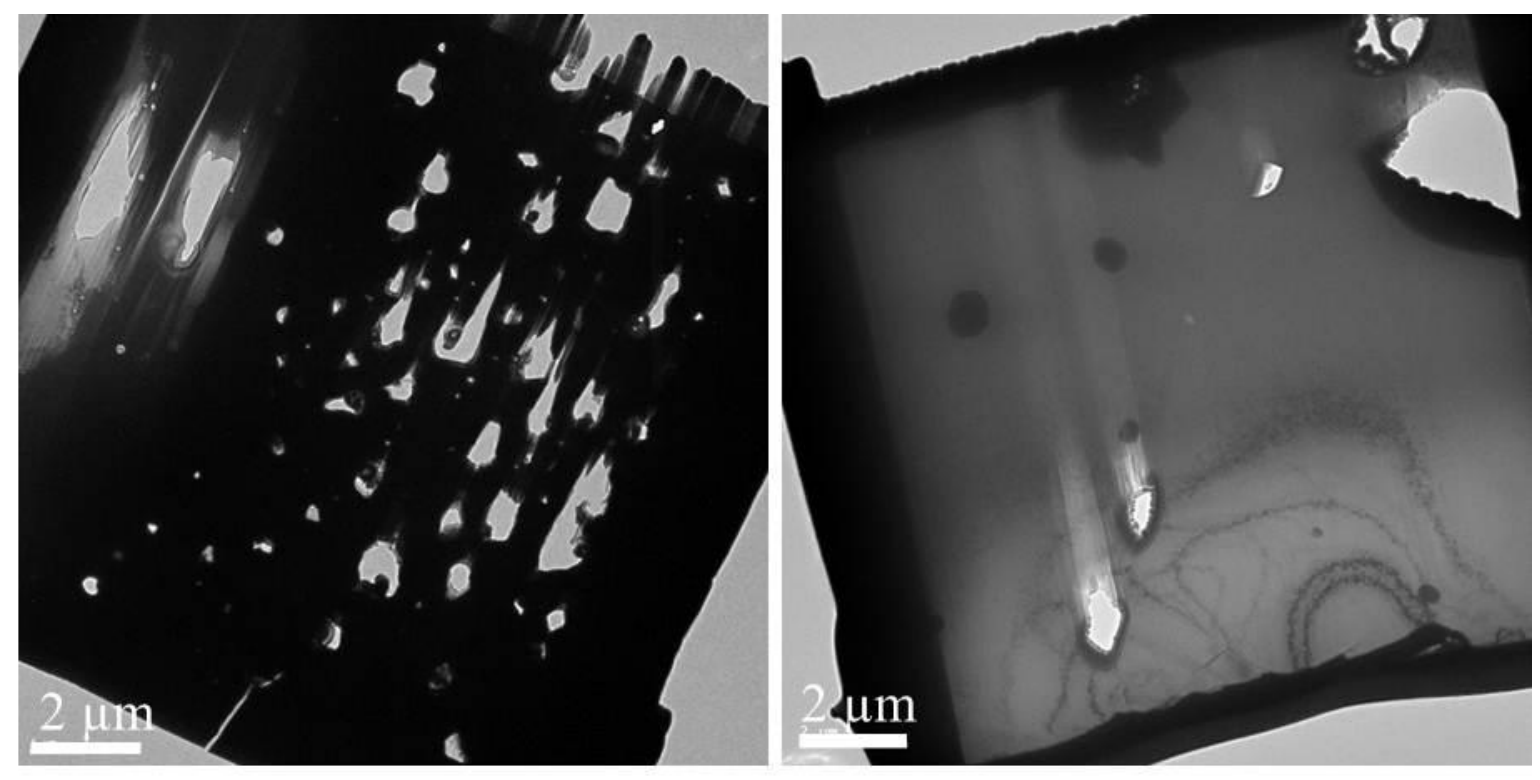

Fig. 8. 